\title{
Factores determinantes para una acción ambiental positiva de la Gestión Integral de Residuos (GIR) en el cantón de Guácimo, Costa Rica
}

\section{Determinants for positive environmental action of the solid waste management of Guacimo, Costa Rica}

Rooel Campos-Rodríguez'

María Marta Camacho-Álvarez²

Fecha de recepción: 16 de mayo del 2014

Fecha de aprobación: 28 de julio del 2014

Campos-Rodríguez, R,; Camacho-Álvarez, M. Factores determinantes para una acción ambiental positiva de la Gestión Integral de Residuos (GIR) en el cantón de Guácimo, Costa Rica. Tecnología en Marcha.Vol. 27, Nº

4, Octubre-Diciembre. Pág 89-101.

I Profesor-Investigador. Escuela de Agronegocios, Instituto Tecnológico de Costa Rica. Costa Rica. Correo electrónico: rocampos@itcr.ac.cr.

2 Directora de la Escuela de Formación Docente, Universidad de Costa Rica. Correo electrónico: mariamarta.camacho@ucr.ac.cr. 


\section{Palabras clave}

Residuos sólidos; Municipalidad de Guácimo; acción ambiental positiva.

\section{Resumen}

En este artículo se presentan los resultados de un estudio sobre los conocimientos, actitudes y barreras en el proceso de implementación de un sistema de gestión integral de residuos sólidos (GIR) en el cantón de Guácimo, Limón, Costa Rica. La municipalidad, como institución rectora del cantón en este tema, no cuenta con un estudio de estas características, de manera que esta investigación será un punto de partida para recabar información de línea base y encaminarse hacia la GIR.

Para alcanzar el objetivo se desarrollaron, validaron y aplicaron dos encuestas, una entre los miembros del Concejo Municipal y viviendas seleccionadas por barrios según sus ingresos económicos, de acuerdo con el reglamento de estudios de composición del país; y otra en locales comerciales escogidos y clasificados por sus actividades según el Código Industrial Internacional Uniforme (CIIU). En todos los casos se aplicaron herramientas estadísticas para la selección de la muestra y comparación de los datos. Una vez capturada la información, se sistematizó y organizó en atributos tales como conocimientos, actitudes, comportamientos, valoraciones y barreras para la GIR en Guácimo.

En la población de Guácimo existe un descontento generalizado por el accionar de las autoridades locales en el tema de la GIR, y a la vez existe un compromiso manifiesto del Concejo Municipal y los demás actores sociales para dirigir el cantón hacia un desarrollo sostenible, que involucre el aprovechamiento, tratamiento y disposición adecuada de los residuos sólidos.

Se espera que la información obtenida en esta investigación le sirva a la Municipalidad de Guácimo para tomar decisiones públicas sostenibles, que involucren a la comunidad en un trabajo colaborativo para que gestionen adecuadamente los residuos sólidos del cantón.

\section{Key words}

Solid waste; Guácimo municipality; positive environmental action.

\section{Abstract}

The aim of this paper is to show the results of a study on knowledge, attitudes and barriers to implement a comprehensive solid waste management program (SWMP) in the canton of Guácimo, Limón, Costa Rica. The municipality, as the governing institution of the county, does not possess a study of this nature, so this research will be a starting point to gather baseline information and move towards the SWMP.

In order to achieve the objective, two surveys were created, validated and applied to the municipal council; the selected neighborhoods according to their income levels, according to the regulations of composition studies in the country, and selected businesses ranked according to their activities as classified in the ISIC (International Standard Industrial Code).

In all cases statistical tools for the sample selection and data comparison were applied. Once data was collected, it was systematized and organized in attributes such as knowledge, attitudes, behaviors, values and barriers to SWMP in Guácimo.

There is a public dissatisfaction on Guácimo municipal actions in terms of a SWMP, while there is a clear commitment from the same city council and other social actors to route the canton towards sustainable development, involving correct use, proper treatment and disposal of municipal solid waste.

It is expected that the information obtained in this research will help the municipality of Guácimo to take sustainable decisions involving the community in a collaborative effort to properly manage solid waste in the Canton. 


\section{Introducción}

Este artículo está basado en la investigación "Actualidad y perspectivas futuras de la gestión de los residuos sólidos en el cantón de Guácimo, Limón, Costa Rica" (Campos, 2014) y expone aspectos relacionados con los conocimientos, actitudes, comportamientos y barreras de un grupo de estudio respecto a la Gestión Integral de los Residuos Sólidos (GIR) de su comunidad.

Los residuos sólidos son un problema mundial y como ejemplo se pueden citar algunos acontecimientos en México, Venezuela, Argentina y Costa Rica. Autores como Rivera (20/3) y Taboada, Aguilar, Ojeda y Cruz (2013) manifiestan que en la ciudad de México se producen cerca de 30,000 toneladas de residuos sólidos urbanos, en su mayoría material valorizable, que terminan, en el mejor de los casos, en un relleno sanitario. Una situación similar se vive en Venezuela, donde los residuos valorizables se pierden entre toneladas de basura en los rellenos sanitarios (Medrano 20l3).

Otro caso se presenta en la provincia de Mendoza, Argentina, que depende del riego artificial por medio de canales abiertos que colapsan por la acumulación de envases tipo PET (Rauek, Torchia y Salomón, 2013).

En el caso de Costa Rica la situación es similar. Campos y Soto (2014) mencionan la poca valorización de los residuos en algunas zonas del país, donde se entierran toneladas de materiales aptos para continuar su ciclo de vida en los procesos productivos. Todo esto acompañado de hábitos de consumo de la población que impactan directamente en la composición de los residuos sólidos, además de la falta de un compromiso serio por parte de los actores involucrados, ya que los ciudadanos generan los residuos esperando que el municipio se haga cargo de ellos.

Por otra parte, en general no existe una cultura integral desde la perspectiva de la planificación, la educación ambiental formal, no formal o comunitaria que contribuya a una conducta pro ambiental de los ciudadanos, a través del desarrollo de habilidades, destrezas, conocimientos y tecnologías para generar una menor cantidad de residuos, así como disponer de la mejor manera de los que se produzcan. Este tipo de cultura generaría un proceso de sensibilización y empoderamiento, que derivaría en el apropiamiento y aplicación de los conceptos teóricos que se brindan en la educación ambiental sobre el tratamiento de estos desechos.

Esta situación evidencia la necesidad de promover una conducta pro ambiental entre los ciudadanos, con el fin de lograr el uso racional de los recursos (Corral y Queiroz, 2004).

Si un individuo puede desarrollar la capacidad de reflexión sobre su propia conducta, podría observar de forma más imparcial sus propios valores y la forma en que estos afectan a otras personas y al planeta (Caduto, 1992).

Se requieren cambios de actitud y hasta de estilos de vida para detener el deterioro ambiental. Es por ello que para establecer programas de educación ambiental es necesario contar con estudios de información primaria sobre los aspectos que influyen en la adopción de una conducta respetuosa del medioambiente (González, 2002).

\section{Metodología}

\section{Área de estudio}

El estudio se realizó en el cantón de Guácimo, provincia de Limón, Costa Rica, cuyas coordenadas geográficas medias son 10 $12^{\prime}$ I $3^{\prime \prime}$ latitud norte y 8337'30" longitud oeste. El cantón cuenta con $576,48 \mathrm{~km}^{2}$ de extensión y está conformado por cinco distritos: Guácimo, Mercedes, Pocora, Río Jiménez y Duacarí, con una población de 41,266 habitantes, según el Instituto Nacional de Estadísticas y Censos (INEC, 20I I).

\section{Herramientas para recolectar la informacion}

Se elaboraron dos encuestas, la primera para su aplicación en una muestra de viviendas seleccionadas y a los miembros del Concejo Municipal de Guácimo; la segunda se aplicó a comercios seleccionados.

Las encuestas contenían preguntas cerradas en su mayoría y algunas abiertas, estas últimas con el propósito de rescatar aspectos generales del sentir de la población sobre el tema de los residuos sólidos. Los instrumentos fueron elaborados, revisados y analizados por un periodo aproximado de cuatro meses por personal de la Municipalidad de Guácimo, la oficina local del Ministerio de Salud, representantes civiles ante la comisión del Programa de Residuos Sólidos (PRESOL) y tres académicos 
expertos en el tema de residuos sólidos, educación ambiental y trabajo comunitario. Después de este proceso las herramientas quedaron validadas para su aplicación. No obstante, también se realizó una prueba piloto en el Parque Central de Guácimo con 20 personas mayores de edad, para corroborar la comprensión de las preguntas y verificar el tiempo estimado de respuesta. Como resultado de las dos validaciones, se hicieron los ajustes. Las encuestas se efectuaron entre el II de febrero y el 23 de abril de 2013.

\section{Criterios de selección y cálculo de la muestra}

Se consideró el criterio de la Municipalidad de Guácimo, el PRESOL, el Ministerio de Salud, la academia y los datos suministrados por el INEC para establecer tres estratos socioeconómicos para el sector residencial (alto, medio y bajo), dos muestras del sector comercial, clasificadas a partir de las actividades que realizaban según el Código Industrial Internacional Uniforme (CIIU), y el sector político de Guácimo (Concejo Municipal) conformado por regidores y síndicos.

Todo ello según los cálculos de muestra realizados por Campos y Soto (2014) para el estudio de generación y composición del cantón de Guácimo, lo que derivó en una muestra de 17| viviendas, 27 del estrato alto (que corresponde al I5\% de la población local), 66 del estrato medio (39\% de la población) y 78 del estrato bajo (46\% de la población), y se encuestó a una persona mayor de edad por vivienda.

Para el sector comercial, la muestra representativa fue de 52 comercios y 41 supermercados, en los cuales se encuestó a la persona encargada. Además se encuestó a 19 representantes del Concejo Municipal.

\section{Prueba estadística}

Una vez capturada la información, se tabularon las preguntas en una hoja de cálculo de Excel, realizando subdivisiones de los siguientes atributos: conocimientos, actitudes, comportamientos, valoraciones y barreras. Conociendo de antemano que se trabajaba con muestras menores a 30 , y que no había normalidad de los datos, se aplicó la prueba no paramétrica de Kruskal-Wallis para determinar la existencia o no de diferencias estadísticamente significativas, utilizado un $p$ value de 0,05. También se utilizó la prueba de Chi Cuadrado para determinar la dependencia de los atributos con los estratos. Para esto se utilizó el programa Infostat.

\section{Resultados y discusión}

A continuación se muestran los resultados más relevantes de las comparaciones realizadas entre los estratos analizados.

\section{Generalidades}

Respecto a los encuestados de las viviendas, el 83\% eran mujeres, $95 \%$ costarricenses, todos mayores de edad, la mayoría en un rango superior a los 34 años (7I\%), con un $12,75 \%$ de personas con estudios universitarios completos o incompletos, el 39,44\% con secundaria completa o incompleta y el $47,81 \%$ con primaria completa o incompleta.

Para el caso del sector comercial, el 52\% de los encuestados eran mujeres, el $90 \%$ costarricenses y todos mayores de edad.

El Concejo Municipal de Guácimo estaba constituido en un 58\% de mujeres, el 100\% costarricenses, todos mayores de edad, ubicándose la mayoría de los encuestados en una edad superior a los 42 años (84\%), con un $26 \%$ de personas con estudios universitarios completos o incompletos y el grueso de los entrevistados (63\%) con educación primaria completa o incompleta.

\section{Conocimientos de la población sobre residuos sólidos}

Caduto (1992) propone que si un individuo puede desarrollar una capacidad de autorreflexión sobre su propia conducta, podría observar de forma más imparcial sus propios valores y la forma en que estos afectan a otras personas y al planeta.

Para llegar a esta conducta ambiental es necesario tener la habilidad de comprender y evaluar el impacto que tiene la sociedad sobre el ecosistema, así como los orígenes, implicaciones y consecuencias de los diferentes problemas ambientales, es decir, se necesita conocimiento ambiental (Mata, Zúñiga, Brenes, Carrillo, Charpentier, Hernández y Zúñiga, 2003).

Tener conocimiento ambiental influye en la definición o redefinición de los conceptos ambientales para poder poner en práctica procedimientos y ser capaces de realizar acciones a favor del ambiente y sensibilizar las actitudes positivas (Mata et al., 2003). 
A los encuestados se les consultó sobre sus conocimientos de la ley 8839 de gestión integral de residuos (GIR), la cual dicta una serie de responsabilidades y derechos a los municipios y ciudadanos. Para encaminarse en la ruta de la GIR es necesario que el costarricense conozca esta ley y la aplique. El 36,84\% de los entrevistados del Concejo Municipal dijo conocer la ley, mientras que en las viviendas el grado de conocimiento no llega al $4 \%$.

La llamada ley GIR también propone la característica de valoración, es decir, la capacidad de aprovechamiento de un residuo, razón que lo separa de un desecho o basura. En la ley, la jerarquía de los residuos sólidos se propone como otro tema importante, de forma tal que la población en general conozca que el reciclaje, aunque es una opción viable, no constituye lo primero, sino el evitar la adquisición de productos con alto potencial de contaminación ambiental. En términos generales, los conocimientos sobre el concepto de residuo y jerarquía son bajos entre las viviendas y el Concejo Municipal (menos del 20\% en todos los casos).

En lo que respecta a los comercios, supermercados y el Concejo Municipal, no existen diferencias estadísticamente significativas $(p>0,05)$ en este punto. Los conocimientos sobre la ley GIR no alcanzan el $6 \%$ en los comercios, mientras que 10 de cada 100 personas, tanto para comercios como para supermercados, dicen conocer lo que es la jerarquía de los residuos sólidos. Cerca del 69\% de toda la muestra dice conocer cómo separar los residuos sólidos. En el cuadro I se presentan los resultados obtenidos.

En el cuadro anterior se observa que los encuestados tienen el conocimiento para separar los residuos pero no lo llevan a la práctica, ya que ante preguntas de corroboración, manifiestan que los residuos se mantienen en su hogar o comercio en una misma bolsa, sin separar, hasta que pase el camión recolector. Esto también se evidencia en los datos que suministran Campos y Soto (2014), ya que manifiestan que el $35 \%$ de los materiales que son colocados en las calles de Guácimo por los ciudadanos para ser transportados mediante el camión recolector son valorizables y el $45 \%$ biodegradables, lo que significa que el $80 \%$ del material que se está desechando en la basura tradicional es susceptible de valoración.

Al aplicar las respectivas pruebas estadísticas, se determina una relación entre los conocimientos y el estrato, dado que el Concejo Municipal presenta mayores conocimientos, pero no existen diferencias significativas entre las respuestas de los seis estratos, por lo que hay evidencia estadística para decir que los conocimientos del área de estudio son similares.

\section{Actitudes de la población con respecto la gestión de los residuos sólidos}

Las actitudes de las personas hacia la GIR constituyen elementos importantes para definir el comportamiento. Por tal motivo, deben ser tomadas en cuenta en los procesos educativos dada su repercusión en la transformación de la cultura. Una actitud constituye una organización relativamente duradera de creencias en torno a un objetivo o situación que predispone a la persona a responder de determinada manera ante ese objeto o situación (Caduto, 1985).

En esta investigación, las actitudes se determinaron por el grado de anuencia de los encuestados a participar en proyectos ambientales, jornadas de capacitación, comisiones cantonales, planes de GIR,

Cuadro I. Conocimientos sobre algunos temas de residuos sólidos en la muestra de estudio (en porcentaje).

\begin{tabular}{|l|c|c|c|c|c|c|}
\hline & \multicolumn{7}{|c|}{ Estrato de estudio } \\
\hline \multicolumn{1}{|c|}{ Conocimiento } & Alto & Medio & Bajo & Comercios & Supermercados & Concejo Municipal \\
\hline Ley 8839 & 0,00 & 3,03 & 3,85 & 5,88 & 4,88 & 36,84 \\
\hline Concepto residuo & 0,00 & 16,67 & 8,97 & 17,65 & 9,76 & 17,65 \\
\hline Jerarquía residuo & 7,41 & 10,61 & 12,82 & 9,8 & 9,76 & 10,53 \\
\hline Separación residuos & 73,08 & 68,52 & 58,93 & 68,29 & 69,23 & 68,42 \\
\hline
\end{tabular}


así como su disposición a pagar más por la recolección, separar los residuos sólidos si hubiera recolección por separado y el grado de responsabilidad de cada uno por la GIR en Guácimo. En la figural se observan los resultados.

Se observa en todos los estratos una actitud positiva respecto a participar en jornadas de capacitación, proyectos ambientales y comisiones cantonales para la GIR.

Además, existe una tendencia por parte del grupo de estudio a no sentirse responsable de la gestión de los residuos sólidos, a excepción del estrato alto, donde un $78 \%$ considera que sí lo es. El resto del grupo, incluso los mismos gestores municipales, ministeriales o políticos, lo ven como una obligación propia del municipio.

Si bien es cierto, la ley señala a la Municipalidad como la responsable de la gestión integral de los residuos generados en su cantón, también es clara en manifestar que el Ministerio de Salud será el rector en materia de gestión integral de residuos, con potestades de dirección, monitoreo, evaluación y control. Además, existe una corresponsabilidad de los productores, importadores, consumidores y gestores de residuos, tanto públicos como privados.

Respecto a la disposición para pagar más por el servicio de recolección y tratamiento de residuos sólidos, cuya tarifa es de US\$2,56 por mes por vivienda, las personas que manifiestan estar de acuerdo estarían dispuestas a pagar un monto adicional de entre US\$I y US\$I,98 por mes.
En el caso de los comercios y supermercados, ante un posible aumento de la tarifa actual de recolección y disposición de residuos (US $\$ 5,12$ por mes), el 20\% de los comercios estaría dispuesto a pagar más por el servicio y los supermercados en un $15 \%$. El monto adicional máximo a pagar sería de US\$1,98 por mes.

Como indican Camposy Soto (20 I 4), la Municipalidad de Guácimo cuenta con cobros diferenciados en la recolección y servicio de limpieza de vías públicas y privadas, pero no dispone del personal suficiente para realizar estas labores ni del equipo para determinar el peso de los residuos sólidos, por lo que solo aplica los cobros más bajos de cada categoría, en este caso US $\$ 2,56$ por mes por vivienda y $\$ 5,12$ por comercio por mes.

Se puede notar una intención de actuar positivamente por parte del sector en estudio, sin embargo, se debe tener claro el hecho de que no porque existan actitudes positivas, eso se reflejará en hechos concretos, porque la actitud no es un análogo del comportamiento, según Charpentier (2004). De igual forma, la misma autora y Mata, Zúñiga, Brenes, Carrillo, Charpentier, Hernández y Zúñiga (2009) señalan que en los programas de educación ambiental, además de las actitudes y el conocimiento, es necesario tomar en cuenta las creencias y los valores, que conducirán a tener un control de lo que se hace (empoderamiento) y así vencer las barreras, y por medio de la autodeterminación trascender del

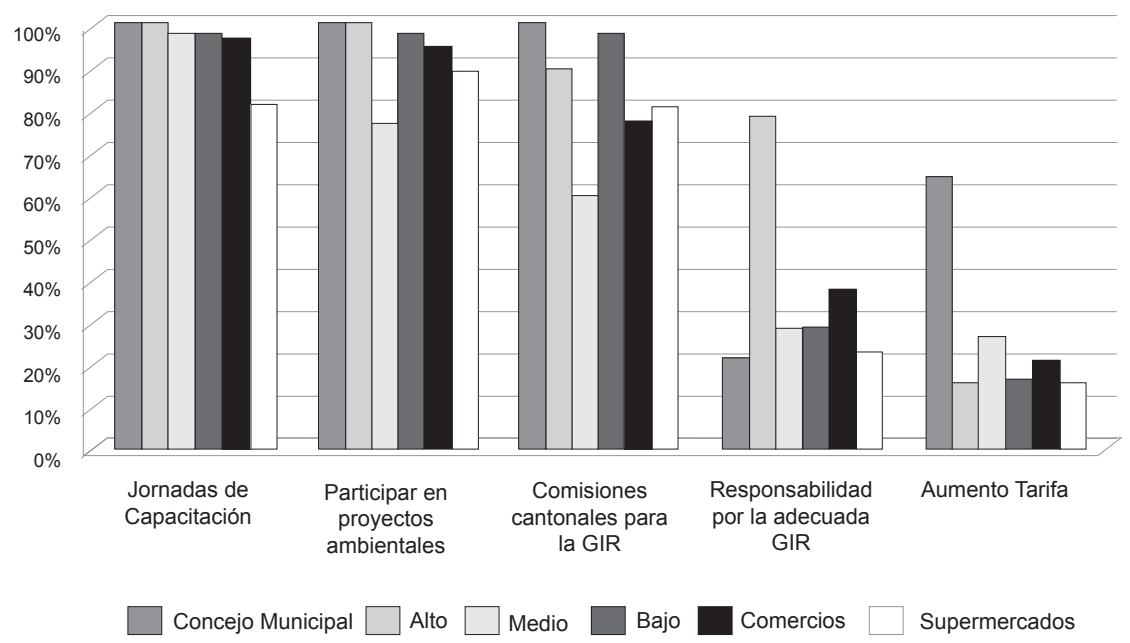

Figura I. Intención de actuar en la muestra de estudio respecto a los residuos sólidos. 
comportamiento ambiental a una acción ambiental positiva.

No existen diferencias significativas entre la intención de actuar en cada uno de los estratos ( $p>0,05)$, pero se presenta una dependencia entre la actitud y el estrato, por lo que ante un eventual proceso de capacitación para la gestión integral de los residuos sólidos en Guácimo se podría esperar una actitud similar en la muestra de estudio.

\section{Comportamiento de la población en relación con la gestión de los residuos sólidos}

Para valorar este atributo las preguntas se dirigieron a conocer si los individuos separaban los residuos, la manera de clasificarlos y la forma de almacenamiento hasta que el camión recolector los recoge.

Al analizar las frecuencias sobre la separación de los residuos para su posterior aprovechamiento, 3 de cada 10 personas del estrato alto dice hacerlo, mientras que el $21 \%$ y $17 \%$ de los estratos medio y bajo, respectivamente, lo hacen. Ante un cuestionamiento para corroborar esta información, tanto el estrato alto como el bajo indican en un 100\% que mantienen los residuos todos juntos en una misma bolsa sin separar, y 8 de cada 10 personas del estrato medio presentan este comportamiento. De esto se puede derivar que, aunque algunas personas por su misma concientización sacan los residuos sólidos separados, los porcentajes de separación son bajos en la muestra estudiada de viviendas en Guácimo, tal y como lo indican Campos y Soto (20 I4), ya que el $80 \%$ de estos residuos son valorizables. Esto mismo han reflejado los estudios de Solís (20 I0), los cuales indican que la mayoría de la población costarricense (57,9\%) no se siente cómoda con la acción de separar los residuos sólidos.

En cuanto al Concejo Municipal, 8 de cada 10 personas dice separar; no obstante, al corroborar la información, el 100\% manifiesta mantener todos los residuos mezclados en un mismo contenedor hasta la llegada del camión recolector, situación que se ajusta más a la realidad del cantón expresada por Campos y Soto (20|4).

Para los comercios y supermercados la situación es similar, ya que los residuos no se separan en la fuente, comportamiento que refleja el estudio de Campos y Soto (20/4) al apuntar que el $88 \%$ del material en los supermercados y un $78,5 \%$ en los comercios se puede valorizar.

Al analizar las relaciones entre los factores y la conducta de separación de residuos sólidos, se observa que no hay diferencias significativas $(p>0,05)$ en el comportamiento de los estratos analizados y tampoco existe relación entre los estratos y el comportamiento, es decir, el comportamiento no depende del estrato para este caso de análisis.

Con toda la información analizada hasta aquí se evidencia que los conocimientos, actitudes y comportamientos de los estratos en estudio son similares. Esto da pie a que se puedan realizar de forma conjunta procesos de educación ambiental para la gestión integral de los residuos sólidos en Guácimo, y fomentar el trabajo colaborativo entre los actores involucrados, con el fin de transformar los conocimientos, actitudes y comportamientos ambientales de la población.

\section{Valoraciones de la gestión de los residuos sólidos}

Para este atributo se le pidió a los encuestados que emitieran su punto de vista respecto a dos aspectos. El primero se refirió al servicio municipal en general, y se tomaron en cuenta la frecuencia de recolección, el cumplimiento de horarios, la recolección de todos los residuos, el ruido emitido por los camiones recolectores, los residuos que quedaban en las calles después de que había pasado el camión recolector, los lixiviados provenientes de los camiones y la forma de conducir de los choferes municipales. Como segundo punto, se analizó la valoración del contexto tomando en cuenta aspectos tales como presencia de malos olores, aumento del dengue, personas que abren las bolsas de basura, acumulación de residuos en las aceras antes de la recolección, botaderos clandestinos, animales que rompen las bolsas de basura y presencia de desechos por varios días en las calles.

\section{Valoraciones del servicio municipal}

El cuadro 2 y la figura 2 evidencian que el cumplimiento del horario del camión recolector no es considerado positivo por los encuestados, ya que a lo sumo 37 de cada 100 personas dicen estar de acuerdo en que se cumple. En términos generales, los estratos analizados también consideran que la recolección de basura una vez por semana no es suficiente. Por otra parte, a excepción del estrato 
Cuadro 2.Valoración sobre el servicio de recolección por parte de la muestra de estudio.

\begin{tabular}{|c|c|c|c|c|c|c|}
\hline & \multicolumn{6}{|c|}{ Estrato en frecuencias relativas } \\
\hline Valoración & $\begin{array}{c}\text { Concejo } \\
\text { Municipal }\end{array}$ & Alto & Medio & Bajo & Comercios & Supermercados \\
\hline $\begin{array}{c}\text { Ruido excesivo camiones } \\
\text { recolectores }\end{array}$ & 47 & 85 & 71 & 96 & 27 & 54 \\
\hline Basura tirada & 74 & 85 & 74 & 97 & 53 & 46 \\
\hline $\begin{array}{c}\text { Presencia lixiviados } \\
\text { Conducción inadecuada } \\
\text { de camión recolector }\end{array}$ & 47 & 63 & 88 & 82 & 90 & 50 \\
\hline
\end{tabular}

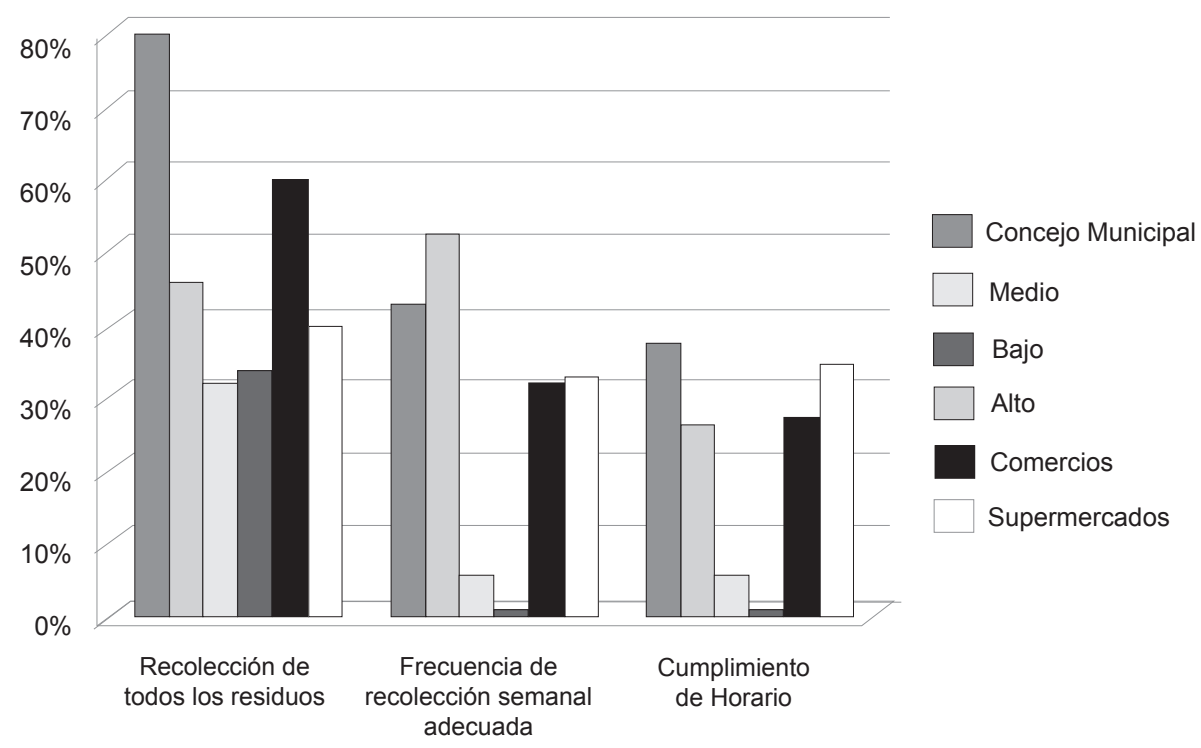

Figura 2.Valoración respecto a los trabajos de recolección de residuos sólidos.

político, todos los demás no consideran que se dé una recolección total de todos los residuos.

Es meritorio mencionar que los estratos analizados consideran que la forma de conducir el camión recolector por parte de los choferes municipales es adecuada.

Todo lo anterior coincide plenamente con lo expuesto por Campos y Soto (20I4), cuando se refieren a que la Municipalidad de Guácimo maneja sus residuos sólidos con poca planificación, hay incumplimiento de horarios y rutas de recolección y acumulación de basuras en calles y aceras, lo cual en términos generales indica un servicio de mala calidad.
Existe una relación entre la valoración del servicio municipal y el estrato, además se evidencian diferencias significativas $(p>0,05)$, dado que los estratos alto y bajo califican de forma más deficiente el servicio municipal respecto a los otros estratos.

Los comercios y supermercados no presentan diferencias significativas $(p>0,05)$.

\section{Valoraciones del contexto}

El cuadro 3 muestra la existencia de basura tirada después que pasa el camión recolector. De igual forma, los desechos permanecen en ocasiones varios días en las aceras sin ser recolectados, provocando, entre otros problemas, que los animales rompan las bolsas y propiciando la aparición de 
vectores que podrían atentar contra la salud pública. Esto también repercute en la existencia de malos olores, situación que probablemente se debe a la descomposición por la recolección tardía o a que los residuos se colocan en el punto de colecta días antes del horario de recolección.

También se presenta la enfermedad del dengue, que se asocia con los problemas de la inadecuada gestión de los residuos, por ejemplo, acumulación de agua en recipientes dispersos. En tal sentido, como mínimo 63 de cada 100 personas por estrato consideran que se ha dado un aumento en la enfermedad del dengue en el cantón, situación que ratifica el Ministerio de Salud, al indicar que para la semana epidemiológica 16 del año 2014, Guácimo se encuentra entre los primeros 10 cantones con más incidencia de dengue del país.

En términos generales, la valoración del contexto que realizan todos los estratos deja ver deficiencias en la gestión integral de residuos sólidos.

Los resultados obtenidos en relación con las valoraciones del contexto demuestran que sí dependen del estrato y no evidencian diferencias significativas $(p>0,05)$, por lo que se infiere que es un sentir generalizado de la muestra de estudio, respecto al panorama de una inadecuada gestión de residuos sólidos en el cantón, situación que repercute en la proliferación de enfermedades, presencia de malos olores, animales en las vías públicas comiendo o dispersando basura y la contaminación visual por la presencia de residuos, entre otros.

\section{Barreras en la gestión del residuo sólido}

Una barrera es un obstáculo, real o no, que una persona puede tener. Se pueden clasificar en tres tipos: las barreras conceptuales, por la noción errónea de los objetivos y alcances de la educación ambiental; las actitudinales, formadas por la percepción de que los educadores tienen actividades más importantes que la educación ambiental; y las barreras de control, que surgen por la percepción de falta de oportunidades, conocimientos, recursos y habilidades. Si estas barreras se presentan, entonces actuarán sobre la sensibilización, los conceptos ambientales, las habilidades, procedimientos y el empoderamiento y el resultado es la falta de incorporación de una cultura ambiental (Mata et al., 2009).

En relación con las barreras del cantón de Guácimo para la no separación de los residuos sólidos, la primera que se detecta se clasifica como conceptual, ya que los encuestados indican necesitar conocimientos para separar adecuadamente los residuos sólidos. Esto se refleja en que más de 50 de cada 100 personas en todos los estratos dicen necesitar conocimiento para poder separar adecuadamente los residuos (cuadro 4).

En el mismo cuadro también se evidencia la presencia de barreras actitudinales, como la falta de

Cuadro 3.Valoraciones del contexto respecto a la inadecuada gestión de residuos en Guácimo.

\begin{tabular}{|c|c|c|c|c|c|c|}
\hline \multirow[b]{2}{*}{ Valoración } & \multicolumn{6}{|c|}{ Estrato en frecuencias relativas } \\
\hline & $\begin{array}{l}\text { Concejo } \\
\text { Municipal }\end{array}$ & Alto & Medio & Bajo & Comercios & Supermercados \\
\hline Presencia malos olores & 84 & 44 & 77 & 58 & 45 & 68 \\
\hline Aumento dengue & 89 & 63 & 80 & 85 & 69 & 63 \\
\hline $\begin{array}{c}\text { Acumulación de basura en } \\
\text { aceras }\end{array}$ & 74 & 56 & 65 & 51 & 45 & 63 \\
\hline $\begin{array}{c}\text { Animales que rompen } \\
\text { bolsas }\end{array}$ & 32 & 33 & 74 & 96 & 65 & 88 \\
\hline $\begin{array}{c}\text { Basura mojada durante } \\
\text { varios días }\end{array}$ & 47 & 44 & 79 & 70 & 22 & 39 \\
\hline Contaminación visual & 68 & 30 & 89 & 91 & 35 & 54 \\
\hline
\end{tabular}


motivación, presentándose en más de 60 de cada 100 personas.

El otro tipo de barreras presentes son las de control, donde los encuestados detectan que efectivamente el camión recolector no discrimina, es decir, aunque ellos separen los residuos, los camiones lo recolectan todo junto. También se evidencia la falta de centros de recuperación de materiales. El dinero sigue siendo una barrera de control a considerar en Guácimo, así como la percepción de la necesidad de contar con espacio y recipientes de diferentes colores para hacer la separación.

En la figura 3 se pueden observar otras barreras de control en las que sobresale que la población estudiada tiene presente que el camión municipal recolecta todos los residuos sólidos juntos y al final de cuentas, aunque estos fueran separados en la fuente, terminarán enterrados sin valorizarse. Por tal motivo, es un asunto que se debe estudiar para brindar una alternativa a la comunidad.

Las barreras para no separar sí dependen del estrato y ninguna de ellas (conceptuales, actitudinales y de control) presenta diferencias significativas ( $p>$ $0,05)$, por lo que se podría trabajar con jornadas de capacitación similares, partiendo del hecho de que las barreras, aunque se dan en frecuencias diferentes, no son estadísticamente significativas.

Las barreras de control son más preponderantes que las otras, dado que el desarrollo de conocimientos y habilidades está supeditado a la dotación de tiempo y recurso monetario a los encargados de incorporar la dimensión ambiental en los programas educacionales. Además de esto, las barreras de control actúan sobre el empoderamiento y la pertinencia, puesto que si no están presentes se carecerá de la incorporación de la dimensión ambiental (Charpentier 2004).

Cuadro 4. Barreras para la separación de residuos sólidos.

\begin{tabular}{|c|c|c|c|c|c|c|c|}
\hline \multirow{2}{*}{ Barrera } & \multicolumn{5}{|c|}{ Frecuencia relativa } \\
\hline Conocimiento & Conceptual & 53 & 63 & 85 & 94 & 60 & Concejo \\
Municipal & Alto & Medio & Bajo & Comercios & 59 \\
\hline Motivación & Actitudinal & 63 & 93 & 77 & 100 & 65 & 88 \\
\hline Espacio & Control & 42 & 100 & 67 & 56 & 59 & 66 \\
\hline Dinero & Control & 37 & 89 & 33 & 77 & 43 & 61 \\
\hline
\end{tabular}

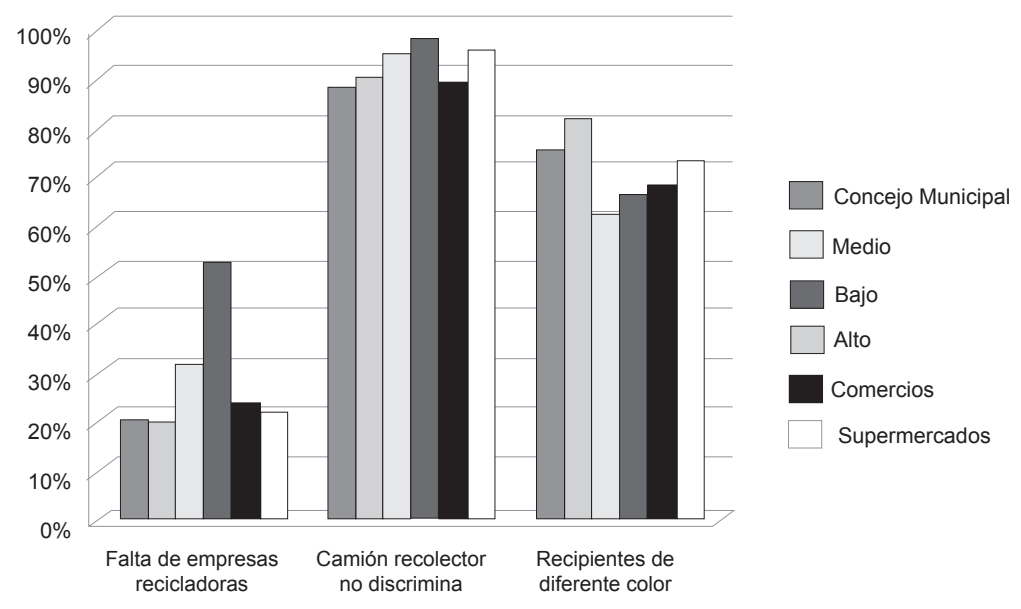

Figura 3. Barreras de control para la no separación de residuos sólidos. 
Tomando en cuenta que las barreras de control son las más importantes ya que influyen sobre el empoderamiento, en la figura 4 se presentan resultados sobre barreras de control para asistir a jornadas de capacitación en temas de residuos sólidos, tales como tiempo, horario y el factor económico. En términos generales, pareciera que el factor tiempo es el más preponderante para los encuestados; no obstante, al aplicar la prueba estadística, las barreras dependen del estrato y no evidencian diferencias significativas $(p>0,05)$, por lo que, desde la perspectiva de planes de capacitación para superar estas barreras, se podría trabajar de forma conjunta con los estratos.

\section{Conclusiones}

Es necesario tomar en cuenta las repercusiones sociales y ambientales del crecimiento económico desproporcionado, ya que todo tiene un límite y se debe buscar un equilibrio basándose en que la educación ambiental debería considerar todo desarrollo y crecimiento desde una perspectiva ambiental (Novo, 2009). Por esto, la educación ambiental debe estimular nuevos conocimientos que generen en los seres humanos una modificación de éstos, habilidades y cualidades, para así incidir directamente en los hábitos de vida y entender a su vez el funcionamiento del ambiente, lo cual se reflejará en las prácticas cotidianas y permitirá establecer conceptos para generar habilidades de forma tal que los individuos se sientan seguros de realizar iniciativas en pro del ambiente y así poder sensibilizarse y adoptar actitudes positivas (Mata et al., 2003).

En tal sentido, es necesario reforzar los conocimientos en Guácimo respecto a la GIR, por medio de un proceso de educación ambiental puesto que estimula la formación de sociedades justas y ecológicamente equilibradas, ya que la preparación para los cambios depende de la comprensión colectiva de la naturaleza sistémica de las crisis que amenazan el futuro del planeta (Novo, 2009).

La investigación evidencia actitudes pro ambientales en la población de Guácimo, que deben concretarse en acciones palpables que coadyuven a la GIR, ya que, como apunta Charpentier (2003), al no ser la actitud un sinónimo de comportamiento, se debe brindar el acompañamiento necesario a la población para que esas buenas intenciones se traduzcan en protección ambiental. Además, se debe tomar en cuenta que las actitudes son estructuras por crear mediante la educación ambiental desde tres pilares, el primero es el cognitivo, que está conformado por las creencias, los conocimientos y las ideas; luego está el afectivo, que debe tomar en cuenta los sentimientos que llevan a valorar la conducta de forma positiva o negativa; y por el último el conductual, que se refiere a la intencionalidad de realizar la acción

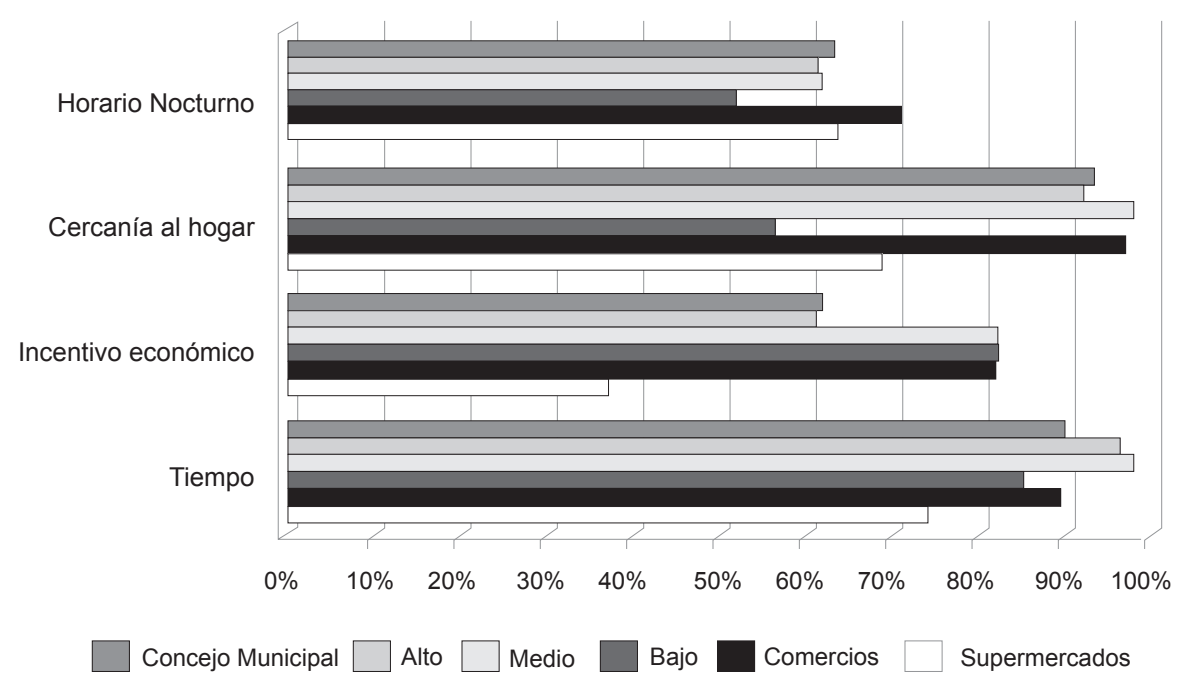

Figura 4. Barreras de control para participar en jornadas de capacitación. 
(Varela, 200 I; Caduto, 1992). Por tal razón, el trabajo de acompañamiento con el grupo de estudio en Guácimo debe darse de forma tal que permita evolucionar desde las buenas intenciones hacia una acción ambientalmente positiva.

Se debe trabajar con la muestra de estudio para superar las diversas barreras respecto a la GIR, ya que si hay presencia de barreras actitudinales, conceptuales y de control, éstas actuarán sobre la actitud, la sensibilización, los conceptos ambientales, habilidades, procedimientos, empoderamiento y pertinencia, lo que no permite incorporar la dimensión ambiental en la GIR de Guácimo.

Tomando en cuenta que la mayoría de los atributos analizados no presentan diferencias estadísticamente significativas, se podría realizar un proceso de educación ambiental con la participación de representantes de todos los actores involucrados, ya que para una gestión integral de residuos sólidos en Guácimo no solo es suficiente contar con elementos técnicos - legales, también es necesaria la incorporación de profesionales y técnicos capacitados en los grupos de trabajo, además de una sociedad informada y sensibilizada, para que la población realice un consumo responsable y participe activamente en las propuestas de la GIR, con instituciones públicas que sean reflejo de un estado comprometido en este tema y agrupaciones sociales que participen con iniciativas y programas específicos (Programa Competitividad y Medio Ambiente, 2008).

Sin embargo, nada de esto será posible sin la participación activa de las comunidades, lo cual hace necesario promover instrumentos metodológicos que fortalezcan y promuevan la capacidad con que cuentan las comunidades para que tengan un control de los procesos a partir de su propia idiosincrasia e interés de transitar por un cambio social, ambiental y cultural (López, López y Rodríguez, 2005).

Por este motivo, la educación ambiental, además de proveer la documentación teórica, debe dar un paso más y procurar que desde las mismas comunidades emanen las alternativas de solución de las problemáticas por medio de modelos educacionales enfocados en una educación ambiental para el desarrollo sostenible y que en este caso particular coadyuven a la gestión integral de los residuos sólidos.
Estos resultados hacen ver la necesidad de tratar la información de forma integral para la toma de decisiones, ya que si el municipio desea proponer procesos de formación que deriven en una gestión adecuada de los residuos sólidos, además de que las personas manifiesten actitudes positivas y se muestren sensibilizadas ante el tema, como indican Mata y colaboradores (2009), es necesario que todos los involucrados tengan la capacidad de saber qué hacer y cómo hacerlo (empoderamiento), y esto se logra en la medida en que se tenga control para emprender una acción y se superen las barreras.

\section{Bibliografía}

Caduto, M. (1985). Guía para la enseñanza de valores ambientales. Vermont, US: Instituto de Ciencias Naturales de Woodstock.

Caduto, M. (1992). Guía para la enseñanza de valores ambientales. Programa Internacional de Educación Ambiental. Madrid: Unesco-Pnuma.

Campos, R. (20 I 4). Actualidad y perspectivas futuras de la Gestión de los Residuos Sólidos en el cantón de Guácimo, Limón, Costa Rica. (Tesis para optar por el grado de Doctorado en Ciencias Naturales para el Desarrollo, no publicada). Instituto Tecnológico de Costa Rica.

Campos, R. \& Soto, S. (20 I4). Análisis de la situación del estado de la Gestión Integral de Residuos (GIR) en el Cantón de Guácimo, Costa Rica. Tecnología en Marcha, 27( I), | | 4- 124.

Charpentier, C. (2004). "Las barreras para la Educación Ambiental pueden superarse". Biocenosis: revista de educación ambiental. Universidad Estatal a Distancia (UNED). San José, Costa Rica, Vol. I 8 ( I-2), I03- 108.

Corral, V. \& Queiroz, J. (2004) Aproximaciones al estudio de la conducta sustentable. Medio Ambiente y comportamiento Humano. Editorial Resma.

González, A. (2002). La preocupación por la calidad del medio ambiente. Un modelo cognitivo sobre la conducta ecológica. (Tesis para optar por el grado de Doctorado en Psicología). Universidad Complutense de Madrid.

INEC. (23 febrero, 20I I). INEC Costa Rica. Obtenido de http:// www.inec.go.cr/Web/Home/GeneradorPagina.aspx.

López, E., López, C. \& Rodríguez, A. (2005). Avances y perspectivas de un modelo de educación ambiental en la zona indígena chontal de Tabasco. México. Obtenido de http://anea. org.mx/eventos/comie2007/Extenso/PonenciaLopezE_ RodriguezA_RicaldeC.pdf

Mata, A., Zúñiga, C., Brenes, O., Carrillo, M., Charpentier, C., Hernández, L. \& Zúñiga, M. (2003). Estrategias innovadoras para la formación inicial de educadores en el campo ambiental. I ed. Cartago: CECC.Vol. 27. Impresora Obando.

Mata, A., Zúñiga, C., Brenes, O., Carrillo, M., Charpentier, C., Hernández, L. \& Zúñiga, M. (2009). Dimensión Ambiental: 
Estrategias Innovadoras para la Formación Docente. San José: CECC.

Medrano, J. (2013). La caracterización de los residuos sólidos en zonas de Páramo: Caso Comunidad de Tuñame, Estado Trujillo, Venezuela. V Simposio Iberoamericano de Residuos Sólidos. Argentina.

Novo, M. (2009). La educación ambiental, una genuina educación para el desarrollo sostenible. Revista de Educación. ISSN 003|-8082. N Extra I. Obtenido de http://dialnet. unirioja.es/servlet/articulo?codigo $=3019430$.

Programa Competitividad y Medio Ambiente (CYMA). (2008). Manual para la Elaboración de Planes Municipales de Gestión Integral de Residuos Sólidos. San José.

Rauek,T.,Torchia, N. \& Salomón, M. (2013). Propuesta de gestión de envases PET post consumo en Mendoza, Argentina.
Mendoza: V Simposio Iberoamericano de Residuos Sólidos. Argentina.

Rivera, C. (20/3). Caracterización de residuos sólidos urbanos potencialmente valorizables en Naucalpán de Juárez, Estado de México. México: V Simposio Iberoamericano de Residuos Sólidos. Argentina.

Solís, M. (2010). Conductas ambientales de separación de desechos sólidos y ahorro de agua en la población de Costa Rica. Revista Costarricense de Psicología, 29(44), 19-34.

Taboada, P., Aguilar, Q., Ojeda, S. \& Cruz, S. (20 I 3). Análisis de la composición de residuos en comunidades rurales mediante un diseño en bloques completos aleatorizados. México: $V$ Simposio Iberoamericano de Residuos Sólidos. Argentina.

Varela, S. (200I). Curso de Psicología Ambiental. Universidad de Barcelona. 